\title{
Prognostic signature associated with radioresistance in head and neck cancer via transcriptomic and bioinformatic analyses
}

\author{
Guo-Rung You ${ }^{1,2+}$, Ann-Joy Cheng ${ }^{1,2,3 \dagger}{ }^{+}$Li-Yu Lee $^{4}$, Yu-Chen Huang ${ }^{5}$, Hsuan Liu ${ }^{1,6,7}$, Yin-Ju Chen ${ }^{8,9}$ and
} Joseph T. Chang ${ }^{3,10^{*}}$

\begin{abstract}
Background: Radiotherapy is an indispensable treatment modality in head and neck cancer (HNC), while radioresistance is the major cause of treatment failure. The aim of this study is to identify a prognostic molecular signature associated with radio-resistance in HNC for further clinical applications.

Methods: Affymetrix cDNA microarrays were used to globally survey different transcriptomes between HNC cell lines and isogenic radioresistant sublines. The KEGG and Partek bioinformatic analytical methods were used to assess functional pathways associated with radioresistance. The SurvExpress web tool was applied to study the clinical association between gene expression profiles and patient survival using The Cancer Genome Atlas (TCGA)-head and neck squamous cell carcinoma (HNSCC) dataset $(n=283)$. The Kaplan-Meier survival analyses were further validated after retrieving clinical data from the TCGA-HNSCC dataset $(n=502)$ via the Genomic Data Commons (GDC)-Data-Portal of National Cancer Institute. A panel maker molecule was generated to assess the efficacy of prognostic prediction for radiotherapy in HNC patients.

Results: In total, the expression of 255 molecules was found to be significantly altered in the radioresistant cell sublines, with 155 molecules up-regulated 100 down-regulated. Four core functional pathways were identified to enrich the upregulated genes and were significantly associated with a worse prognosis in HNC patients, as the modulation of cellular focal adhesion, the PI3K-Akt signaling pathway, the HIF-1 signaling pathway, and the regulation of stem cell pluripotency. Total of 16 up-regulated genes in the 4 core pathways were defined, and 11 over-expressed molecules showed correlated with poor survival (TCGA-HNSCC dataset, $n=283$ ). Among these, 4 molecules were independently validated as key molecules associated with poor survival in HNC patients receiving radiotherapy (TCGA-HNSCC dataset, $n=502$ ), as $\operatorname{IGF1R}(p=0.0454, \mathrm{HR}=1.43)$, LAMC2 ( $p=0.0235, \mathrm{HR}=1.50)$, ITGB1 $(p=0.0336, \mathrm{HR}=1.46)$, and IL-6 $(p=0.0033, \mathrm{HR}=1.68)$. Furthermore, the combined use of these 4 markers product an excellent result to predict worse radiotherapeutic outcome in HNC $(p<0.0001, \mathrm{HR}=2.44)$.
\end{abstract}

Conclusions: Four core functional pathways and 4 key molecular markers significantly contributed to radioresistance in HNC. These molecular signatures may be used as a predictive biomarker panel, which can be further applied in personalized radiotherapy or as radio-sensitizing targets to treat refractory HNC.

Keywords: Head and neck cancer, Radioresistance, Prognosis, IGF1R, LAMC2, ITGB1, IL-6

\footnotetext{
* Correspondence: jtchang@adm.cgmh.org.tw

${ }^{\dagger}$ Guo-Rung You and Ann-Joy Cheng contributed equally to this work.

${ }^{3}$ Department of Radiation Oncology, Chang Gung Memorial Hospital-Linkou,

Taoyuan, Taiwan

${ }^{10}$ Department of Radiation Oncology, Xiamen Chang Gung Memorial

Hospital, Xiamen, Fujian, China

Full list of author information is available at the end of the article
}

(c) The Author(s). 2019 Open Access This article is distributed under the terms of the Creative Commons Attribution 4.0 International License (http://creativecommons.org/licenses/by/4.0/), which permits unrestricted use, distribution, and reproduction in any medium, provided you give appropriate credit to the original author(s) and the source, provide a link to the Creative Commons license, and indicate if changes were made. The Creative Commons Public Domain Dedication waiver (http://creativecommons.org/publicdomain/zero/1.0/) applies to the data made available in this article, unless otherwise stated. 


\section{Background}

Head and neck cancer (HNC) is one of the most prevalent cancers worldwide [1-3]. The standard treatment for patients with $\mathrm{HNC}$ is surgery, radiation, chemotherapy or a combination of these treatments $[4,5]$. Although treatment strategies have advanced in the last two decades, the overall 5-year survival rate for patients has not significantly changed. Tumor recurrence after radiotherapy is a major obstacle to recovery in $\mathrm{HNC}[4,5]$. The identification of radioresistant molecules contributing to a poor prognosis may facilitate patient consulting to determine proper treatment selection to improve the therapeutic outcome.

Previous studies have globally surveyed human genes associated with radioresistance in various cancers. Thus far, different experimental approaches have been used, including the direct comparison of two sets of samples with different levels of radiosensitivity in cancer tissues or cell lines [6-8]. A major disadvantage of this approach lies in the heterogeneity of the samples; the different radiosensitivities among various genetic backgrounds or tissue origins may also have a confounding effect on other pathological phenotypes. The use of microarrays to compare gene expression profiles between parental and radiation-treated cells has also been reported $[9,10]$. Although this approach minimal the cause of genetic variation between individuals, those reports on the cellular and molecular effects often used short-term treatments of irradiation as a research model and may reflect the external radiation-induction situation. To recapitulate the condition of patients with intrinsic radioresistance, we have established several isogenic HNC cell sublines by long-term and low-dose serial irradiation $[11,12]$. These subline cells have been shown possessing higher radioresistant phenotype compared to their parental cells $[11,12]$. Similar approach has also been employed and demonstrated to efficiently generate high radioresistant cells [13-16].

To obtain a more comprehensive profile of the molecular network associated with radioresistance, in this study, we performed cDNA microarrays to globally survey differential transcriptomes between $\mathrm{HNC}$ cells and isogenic radioresistant sublines. We also used bioinformatic software to assess core molecular pathways associated with radioresistance. Through data mining of gene expression profiles available in the public domain, we further identified the biomarker signatures that contribute to the prognosis of HNC. Our study provides predictive or prognostic information, which may be further applied as biomarker criteria regarding treatment choices for individualized therapy in HNC.

\section{Methods}

Establishment of highly radioresistant HNC cell sublines Three HNC cell lines, OECM1, FaDu and Detroit, were used $[12,13]$. The cells were grown in MEM or RPMI
1640 medium supplemented with $10 \%$ fetal bovine serum, as previously described $[12,13]$. The serial irradiation method was used to establish cell sublines with a highly radioresistant capability, similar to that described previously [12]. In principle, the long-term fractionated irradiation method was employed to select radioresistant subclone. Briefly, when cell confluence reached $50 \%$, cells were treated with 2 Gy of radiation and continuously cultured. When they reached to approximate $85 \%$ confluence, the cells were trypsinized and subcultured into new plates. When they grew to $50 \%$ confluence, the cells were treated with another $2 \mathrm{~Gy}$ of radiation. The cells were continuously cultured and repeatedly treated until 60 Gy of irradiation was reached. This method has been previously demonstrated to successfully generate a more radioresistant sublines compared to the parental cells $[11,12]$. In our HNC cells, it took approximately 3 months to complete the irradiation course. These subline cells (OECM1-RR, FaDu-RR, Detroit-RR) were harvested, examined for radiosensitivity, and subjected to cDNA microarray analysis. As shown, all these sublines exhibited higher radioresistance compared to their parent cells (Additional file 1: Figure S1).

\section{Profiling of radioresistant associated genes in HNC cells.}

To globally determine the gene expression profile associated with radioresistance in HNC, cDNA microarray analysis was used to compare the different transcriptomes between $3 \mathrm{HNC}$ parental cell lines and their radioresistant sublines. RNA extraction and cDNA microarray analysis were performed, similarly as previously described [17]. Briefly, total RNA was extracted by TRIzol reagent (Gibco BRL), and the quality and quantity were confirmed using an Agilent 2100 bioanalyzer (Agilent Technologies, Santa Clara, CA). The complementary RNA was hybridized to the Human Affymetrix U133A microarray gene chip and scanned by an Affymetrix GeneArray 2500 scanner (Affymetrix, Santa Clara, CA). Partek Genomics Suite software version 6.6 (Partek, St. Louis, MO) was used to normalize all signal intensities by RMA and to identify the gene expression levels of the microarray data. ANOVA was used to select the genes with a $p$-value $<0.05$ and an average difference in expression of $>1.5$-fold between parental and radioresistant cells. Hierarchical cluster analysis was applied to assess the similarity between sample groups.

\section{Analysis of radioresistant-associated molecular pathways in HNC}

The molecular pathways of the differentially expressed genes identified in the microarray assays were analyzed using the Kyoto Encyclopedia of Genes and Genomes (KEGG) and Partek pathway (Partek, St. Louis, MO) 
analytical methods. KEGG is a collection of databases to provide molecular-level information to understand the functions and utilities of model biological systems [18]. KEGG Orthology groups (KO) analysis was used to identify molecular interaction networks. The computational platform of the Partek pathway algorithm (Partek Genomics Suite software 6.6) was used to identify the significant pathways for the differentially expressed genes according to the KEGG database. The pathways with a $p$-value $<0.05$ were selected as statistically significant candidates.

\section{Identification of radioresistance-associated genes that contribute to a poor prognosis in HNC patients}

The prognostic effect of radioresistance-associated genes in HNC patients was evaluated using the SurvExpress web tool [19]. SurvExpress is a versatile online biomarker validation tool to assess the significance of multi-gene expression in various cancers using survival analysis. The dataset of The Cancer Genome Atlas (TCGA) head and neck cancer cohort $(n=283)$ was used in this study. For SurvExpress analysis, high- and low-risk groups were classified by the optimization algorithm from the order of the prognostic index according to each gene expression level. The high-risk group was selected to perform Kaplan-Meier analysis for overall survival, and the log-rank test was used to calculate hazard ratios (HRs) and their 95\% confidence intervals (CIs).

To further validate the prognostic value of the genes in $\mathrm{HNC}$ patients receiving radiotherapy, a larger-scale, head and neck cancer cohort $(n=502)$ was used. The gene expression levels of the TCGA-HNSCC cohort (level 3, RNASeqv2 RSEM genes normalized data) were obtained from Broad GDAC firehose (gdac.broadinstitute.org). Clinical information about this cohort was retrieved from the Genomic Data Commons (GDC)-Data-Portal of National Cancer Institute (portal.gdc.cancer.gov). In this cohort, $335 \mathrm{HNC}$ patients who received radiotherapy were recruited for prognostic analyses by using log-rank test and KaplanMeier survival method.

To extend the potential application of the candidate molecules, the prognostic significance of the combined markers was further evaluated. The cutoff point of high or low- expression was defined as the medium expression value of each candidate molecule. The highrisk group was defined as the patients who possessed at least one markers with high-expressions, while low-risk group being the patients without any high-level marker. The log-rank test and Kaplan-Meier survival method were used to examine prognostic significance. All $P$ values were two-sided, and the significance level was set at $P<0.05$.

\section{Results}

Gene expression profile associated with radioresistance in HNC cells

To obtain a comprehensive profile of molecules that may represent the intrinsic factor of radioresistance in $\mathrm{HNC}$, we established 3 radioresistant (RR) sublines derived from $\mathrm{HNC}$ cancer cell lines. The cDNA microarray database was established by comparing the mRNA expression profiles between the parental HNC cells and RR subline cells. After ANOVA by the criteria of a $p$-value $<0.05$ and average expression change $>1.5$-fold between parental and RR sublines, 255 genes (294 probe-sets) were filtered out. Unsupervised hierarchical clustering analysis of these 255 genes classified the cells into two groups (parental and RR), in which 155 genes were up-regulated and 100 were down-regulated in the RR sublines (Additional file 1: Figure S2). Additional file 2: Table S1 and Table S2 list the top 100 up-regulated and top 50 down-regulated genes differentially expressed in the RR cells. These genes represent the expression profile associated with radioresistance in HNC cells.

\section{Core molecular pathways associated with radioresistance in HNC cells}

To obtain a global picture of the molecular pathways that may contribute to radioresistance, the 155 up-regulated and 100 down-regulated genes were imported into the KEGG KO suite for integrated network analysis. With the association of the up-regulatory mechanism, these 155 molecules were found to be enriched in the pathways related to oncogenic functions in general. Among the top 10 significant pathways, cell mobility comprised 3 , as the regulation of focal adhesion, extracellular matrix (ECM), and cytoskeleton. Other oncogenic mechanisms, including the PI3K-Akt signaling pathway, HIF-1 signaling pathway, and pluripotency of stem cells, were also noted (Fig. 1a). Regarding the association of a down-regulatory mechanism, 100 molecules were found to be enriched in the pathways mostly associated with cellular metabolism. These include the TCA cycle, proteoglycans in cancer, carbon metabolism, and arachidonic acid metabolism (Fig. 1b). To identify the molecular signature contributing to radioresistance in $\mathrm{HNC}$, the oncogenic associated pathways were addressed, as the modulation of focal adhesion $(p=0.00005)$, PI3K-Akt signaling $(p=0.00007)$, HIF-1 signaling $(p=0.00223)$, and pluripotency of stemness $(p=0.02831)$.

\section{Prognostic significance of the 4 core pathways in HNC patients}

The prominent molecules associated with radioresistance were focus on the 4 core oncogenic pathways. Among the 155 up-regulated genes, 11, 14, 5, and 5 


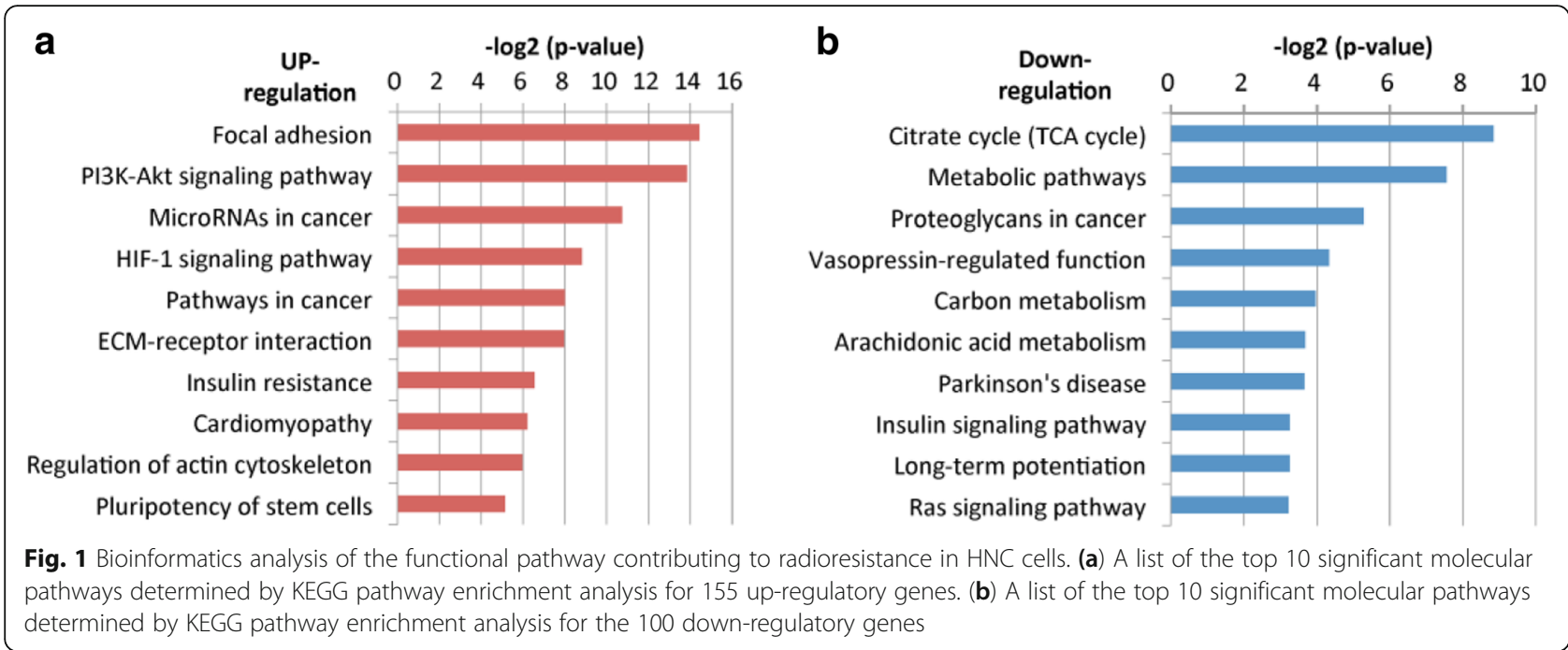

molecules were enriched in the focal adhesion, PI3KAkt, HIF-1, and stemness pathways, respectively (Table 1). Aside from the overlapped molecules, 16 RR-associated genes existed in these core pathways, indicating the high significance of these genes to facilitate radioresistance in $\mathrm{HNC}$.

To determine the clinical significance of these core pathways, we investigated the association between the gene expression levels of these 16 molecules in HNC patients (grouping into 4 specific pathways) and the patient survival status. The TCGA cohort (HNC, $n=$ 283) was used via the SurvExpress analytical method. The results are shown in Fig. 2. As shown, all the core pathways exhibited a prominent correlation with poor survival, as the focal adhesion $(p<0.00001, \mathrm{HR}$ $=2.45)$, PI3K-Akt signaling $(p<0.00001, \quad \mathrm{HR}=2.95)$, HIF-1 signaling $(p<0.00349, \mathrm{HR}=1.93)$, and pluripotency of stem cells $(p=0.00013, \mathrm{HR}=2.03)$. These results suggested that these RR-associated core pathways could distinguish among patients in the high-risk group and predict a poor prognosis in HNC.

Table 1 List of up-regulated genes enriched in the four core molecular pathways

\begin{tabular}{|c|c|}
\hline KEGG pathway name & Gene list \\
\hline Focal adhesion & 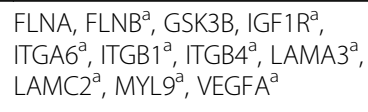 \\
\hline PI3K-Akt signaling pathway & 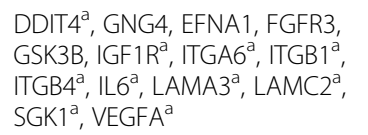 \\
\hline
\end{tabular}

HIF-1 signaling pathway ENO2 ${ }^{a}, I G F 1 R^{a}, I L 6^{a}, S L C 2 A 1^{a}, V E G F A^{a}$

Pluripotency of stem cells FGFR3, FZD10 ${ }^{a}$, GSK3B, IGF1R ${ }^{a}$, JARID2 ${ }^{a}$

${ }^{\mathrm{a}}$ Top 100 up-regulated genes
Prognostic significance of the panel of radioresistant genes in HNC patients

To assess the clinical significance of the 16 RR-associated genes in $\mathrm{HNC}$, we investigated the association between each gene expression and patient survival status using the TCGA cohort (HNC, $n=283$ ) and SurvExpress analytical method. For each gene, the $p$-value and HR are summarized in Table 2. As shown, 11 of the genes exhibited over-expression in the cancer tissues and were statistically correlated with poor survival $(p<$ 0.05): IGF1R, LAMC2, ITGA6, ITGB1, ITGB4, LAMA3, IL-6, DDIT4, SLC2A1, ENO2, and FZD10 (Fig. 3). Thus, this panel of molecules represents molecular biomarkers to predict a poor prognosis in HNC patients.

\section{Prognostic signature for the prediction of a worse radiotherapeutic outcome in $\mathrm{HNC}$}

To further confirm the prognostic value of the RR genes in the patients who received radiotherapy, an independent validation study was performed. Of the 11 genes that exhibited significant values in the prediction of a poor prognosis, 7 involved in more than 2 core pathways (Table 2) were selected. To fast examine whether these 7 molecules screened from the smaller TCGA-HNSCC dataset $(n=283)$ may also exhibit significance in a larger TCGA-HNSCC dataset $(n=502)$, the combinational analysis of these markers with the survival status of the HNC patients were examined via SurvExpress analytical method. As shown, remarkable associations of these combine molecules in either TCGA-HNSCC datasets were found (Additional file 1: Figure S4). Thus, although different approaches may produce various results, the most prominent molecules usually still come out.

In the larger TCGA-HNSCC dataset $(n=502), 335$ patients who received radiotherapy were filtered out for validation study via Kaplan-Meier survival analytical 


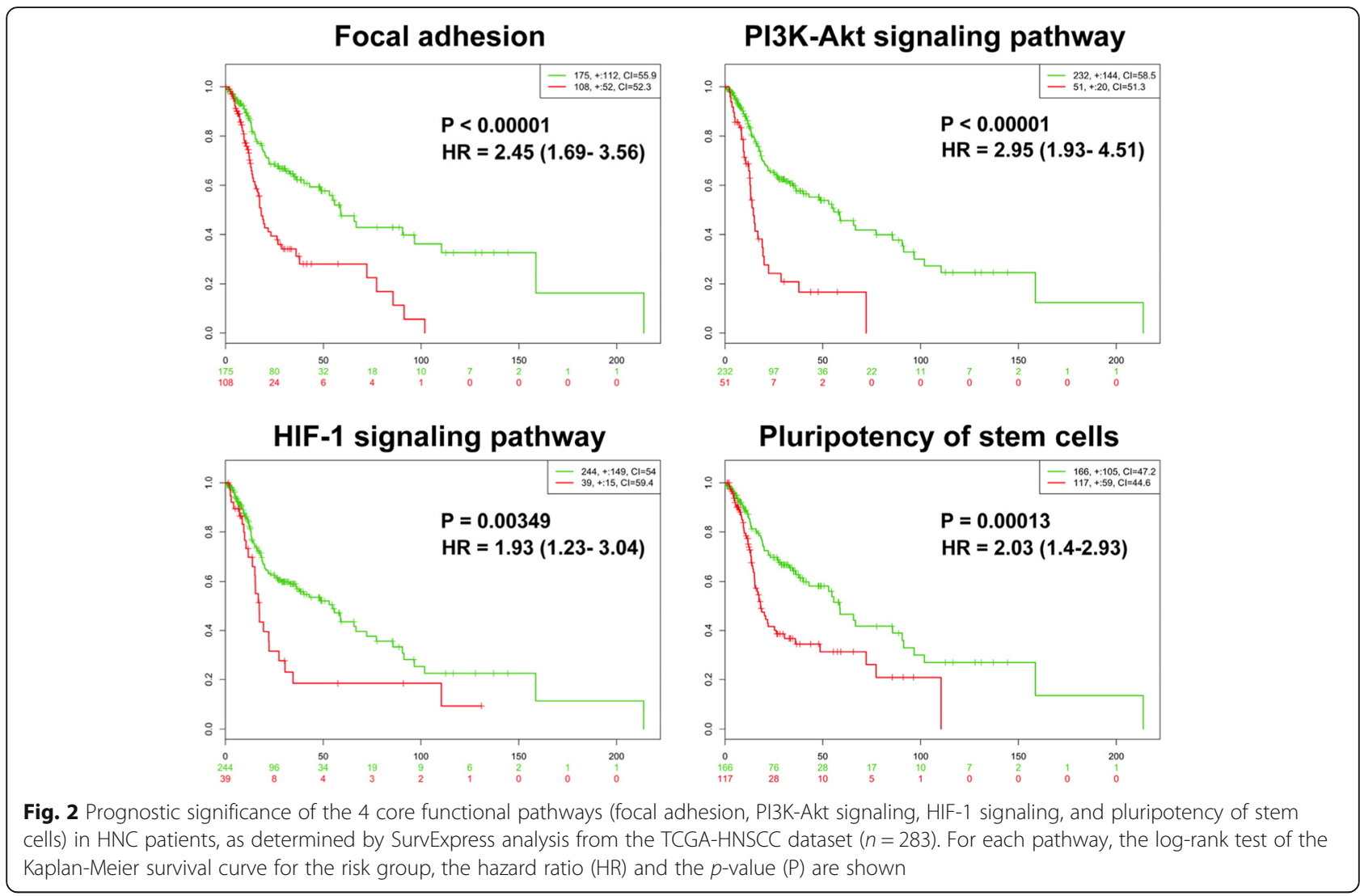

method. As shown in Fig. 4, four molecules were significantly correlated with a poor overall survival, as IGF1R $(p=0.0454, \mathrm{HR}=1.43)$, LAMC2 $(p=0.0235, \mathrm{HR}=1.50)$, ITGB1 $(p=0.0336, \mathrm{HR}=1.46)$, and IL-6 $(p=0.0033$, HR $=1.68$ ). Consistently, these four molecules were confirmed up-regulated in the cellular RR sublines compared to their parental HNC cell lines (Attached file 1: Figure S3). Although ITGA6, ITGB4, and LAMA3 showed no statistical association with patient survival $(p>0.05)$, these molecules were found to be highly correlated with other significant molecules (Additional file 2: Table S3). Thus, these molecules may also play important roles in facilitating radioresistance in HNC.

To enrich potential application of these 4 molecules, the effectiveness of prognostic prediction by the use of combined markers was further determined. For those $335 \mathrm{HNC}$ patients receiving radiotherapy, 277 patients possessing at least one high-level marker was defined as high-risk group, while 58 without any high-level marker was defined as low-risk group. The Kaplan-Meier survival analysis was used to assess the prognostic significance. As shown in Fig. 5, this combined panel showed an excellent association with poor survival $(p<0.0001$, $H R=2.44)$. This result suggested that the use of combined molecules gained an advantage of the individual marker to produce an outstanding prognostic efficacy.
In all, we have identified 4 prognostic biomarkers, IGF1R, LAMC2, ITGB1, and IL-6, and demonstrated a combine panel of molecular signature to predict a worse radiotherapeutic outcome in HNC.

\section{Discussion}

Radiotherapy is an indispensable treatment modality in $\mathrm{HNC}$, while radioresistance is the major cause of treatment failure. Therefore, the identification of resistant molecules may allow further clinical applications in personalized radiotherapy. For this goal, we have obtained several important findings in this study. (1) Without interference by genetic heterogeneity, 255 genes were identified to be associated with radioresistance in HNC cells, including 155 up-regulated and 100 down-regulated genes. (2) Four core molecular pathways significantly contributed to radioresistance in HNC cell lines and HNC patients: modulation of cellular focal adhesion, the PI3K-Akt signaling pathway, HIF-1 signaling pathway, and pluripotency of stem cells. (3) Eleven molecules were associated with a poor survival in HNC, 4 of which, IGF1R, LAMC2, ITGB1, and IL-6, were key molecules to predict a worse prognosis of radiotherapy. Further validation studies are highly recommended to confirm these results in a subgroup of HNC patients, such as for a specific anatomic subsite, a specific ethic 
Table 2 Prognostic significance of the 16 selected genes in the HNC patients

\begin{tabular}{|c|c|c|c|c|}
\hline No. & Gene & $P$-value & Hazard ratio $(\mathrm{Cl})$ & Pathway \\
\hline 4 & IGF1R & 0.03497 & $1.57(1.03-2.41)$ & $\begin{array}{l}\text { Focal adhesion } \\
\text { HIF-1 signaling pathway } \\
\text { PI3K-Akt signaling pathway } \\
\text { Pluripotency of stem cells }\end{array}$ \\
\hline 3 & VEGFA & 0.14780 & $1.31(0.91-1.88)$ & $\begin{array}{l}\text { Focal adhesion } \\
\text { HIF-1 signaling pathway } \\
\text { PI3K-Akt signaling pathway }\end{array}$ \\
\hline 2 & LAMC2 & 0.00228 & $1.97(1.26-3.07)$ & $\begin{array}{l}\text { Focal adhesion } \\
\text { PI3K-Akt signaling pathway }\end{array}$ \\
\hline 2 & ITGA6 & 0.00514 & $1.76(1.10-2.64)$ & $\begin{array}{l}\text { Focal adhesion } \\
\text { PI3K-Akt signaling pathway }\end{array}$ \\
\hline 2 & ITGB1 & 0.01010 & $1.80(1.14-2.83)$ & $\begin{array}{l}\text { Focal adhesion } \\
\text { PI3K-Akt signaling pathway }\end{array}$ \\
\hline 2 & ITGB4 & 0.01463 & $1.57(1.09-2.27)$ & $\begin{array}{l}\text { Focal adhesion } \\
\text { PI3K-Akt signaling pathway }\end{array}$ \\
\hline 2 & LAMA3 & 0.01549 & $1.69(1.10-2.60)$ & $\begin{array}{l}\text { Focal adhesion } \\
\text { PI3K-Akt signaling pathway }\end{array}$ \\
\hline 2 & IL6 & 0.01611 & $1.57(1.08-2.28)$ & $\begin{array}{l}\text { HIF-1 signaling pathway } \\
\text { PI3K-Akt signaling pathway }\end{array}$ \\
\hline 1 & DDIT4 & 0.00002 & $2.45(1.61-3.72)$ & PI3K-Akt signaling pathway \\
\hline 1 & JARID2 & 0.00212 & $1.91(1.26-2.91)$ & Pluripotency of stem cells \\
\hline 1 & SLC2A1 & 0.00421 & $2.17(1.26-3.73)$ & HIF-1 signaling pathway \\
\hline 1 & ENO2 & 0.00447 & $1.69(1.17-2.44)$ & HIF-1 signaling pathway \\
\hline 1 & FZD10 & 0.03798 & $1.71(1.02-2.87)$ & Pluripotency of stem cells \\
\hline 1 & MYL9 & 0.07662 & $0.65(0.41-1.05)$ & Focal adhesion \\
\hline 1 & FLNB & 0.12240 & $1.33(0.92-1.93)$ & Focal adhesion \\
\hline 1 & SGK1 & 0.03847 & $1.64(1.02-2.63)$ & PI3K-Akt signaling pathway \\
\hline
\end{tabular}

$\mathrm{Cl}$ confidence intervals

patient group, or for the cancers caused by a specific etiology.

Four core pathways played substantial roles related to radioresistance in HNC. In the pathway modulating focal adhesion, 11 RR genes were enriched (Tables 1 and 2). Molecules in the laminin (LAMA3, LAMC2) and integrin (ITGA6, ITGB1, ITGB4) families composed the majority in this pathway, indicating the close link between these signaling pathways and radioresistance. Laminin, a family of extracellular matrix glycoproteins, is the major non-collagenous constituent of the basement membrane. It comprises 3 chains, alpha, beta and gamma, to form various heterotrimeric laminin isoforms [20]. Integrin is a family of transmembrane receptors that facilitates extracellular matrix adhesion. It consists of alpha and beta subunits with several isoforms to form a heterodimeric protein [21]. Although the major functions of laminin and integrin are to maintain the mechanical integrity of the cell membrane and regulate cell mobility, recent studies have shown much more biological roles than what was originally thought. Through the interaction of laminin and its cell-surface receptors, including integrin, this complex protein activates signal transduction pathways that may mediate various cellular functions, including cell proliferation, differentiation, invasion, tumor angiogenesis and metastasis [21-23]. In this study, we further noted that up-regulation of this focal adhesion pathway plays an important role in facilitating radioresistance in HNC (Figs. 1 and 2). Our finding is consistent with recent reports that cell adhesion molecules, such as laminin and integrin family proteins, participate in radioresistance, chemoresistance, or cell survival in several cancers [24-28]. We also demonstrated that LAMC2, a laminin component, and ITGB1, an integrin subunit, exhibit excellent correlation with poor outcome in HNC patients receiving radiotherapy (Figs. 3 and 4). These results are supported by other investigations as well. LAMC2 is over-expressed in several types of cancers, including urothelial, lung, colorectal and head-neck cancers, and is associated with a poor prognosis [29-33]. Similarly, a high level of ITGB1 predicts poor survival in breast and gastric cancers [34-38]. Furthermore, LAMC2 or ITGB1 may serve as therapeutic targets via modulating molecular expression to improve radiosensitivity [39-42]. All these results highlight the importance of the laminin-integrin axis pathway in radioresistance and cancer aggressiveness. 


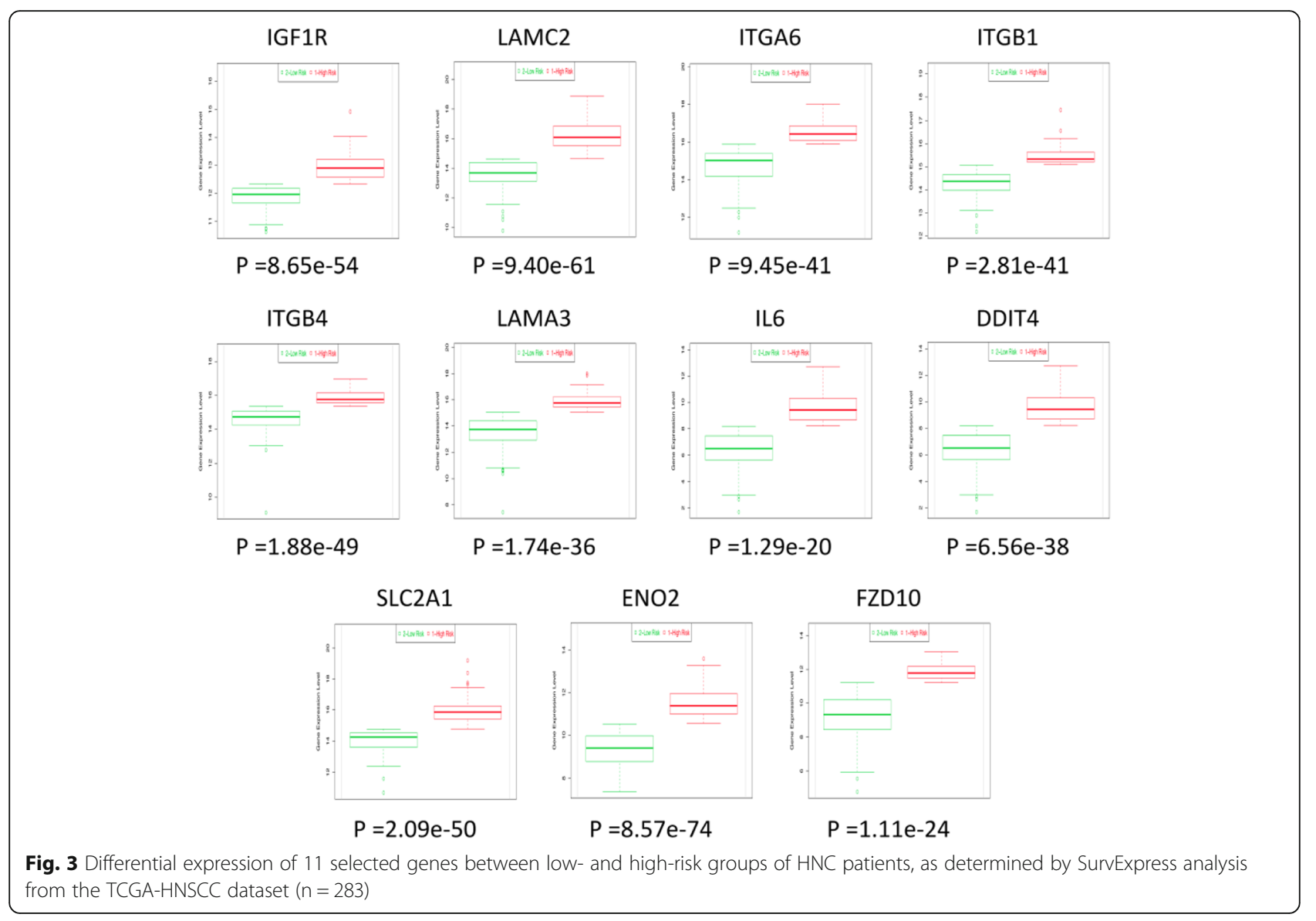

Another pathway identified to be associated with radioresistance, the PI3K-Akt signaling pathway, was shown to be highly significant (Figs. 1 and 2). PI3K-Akt is an intracellular signaling pathway that promotes cell growth and survival in response to extracellular stimuli [43]. It is an important downstream mediator of several membrane-bound receptor tyrosine kinases. Hyper-activation of this pathway correlates with many aggressive cancer phenotypes, such as cell proliferation, tumor angiogenesis, metastasis, and a poor prognosis [43]. Furthermore, the PI3K-Akt pathway contributes to radioresistance in many types of cancers, a finding that was consistent with ours [44, 45]. In this study, we also noted that IL- 6 is a key extracellular signaling molecule participating in the PI3K-Akt signaling pathway (Tables 1 and 2). IL-6 is a pleiotropic cytokine that is involved in multiple biological responses, including auto-immunity, inflammation and cancers [46, 47]. It can mediate numerous downstream effectors by activating several signaling cascades, including the JAK/STAT, MAPK, and PI3K/AKT pathways, to promote cancer progression [47-50]. Specifically, the function of IL-6 in therapeutic resistance has been commonly reported. This molecule can protect cells from radiation- or drug-induced DNA damage by suppressing oxidative stress or the induction of anti-apoptotic mechanism to facilitate cell survival [50-53]. Clinically, IL-6 has also been found to be over-expressed in almost all types of tumors and is associated with a poor prognosis of cancers such as cervical, lung, gallbladder, and head-neck cancers [54-58]. All these reports support our findings that IL- 6 is a critical factor that is over-expressed in cancers and regulates the PI3K-Akt signaling pathway, leading to radioresistance and a worse treatment outcome.

The pathways of cancer stemness and HIF-1 signaling exhibited a prominent function in this study (Figs. 1 and 2). Recently, accumulating studies have shown that cancer stem cells, a subset of cancer cells, possess stem cell-like properties, which may serve as the driving force for tumorigenesis. These cells have a strong malignant potential, with self-renewal capacity, high mobility, and stress tolerance, resulting in resistance to chemoradiotherapy $[59,60]$. The HIF-1 signaling pathway is critical in the maintenance of the cancer stemness phenotype, which may be induced by the hypoxia condition in the tumor microenvironment [61-63]. This 

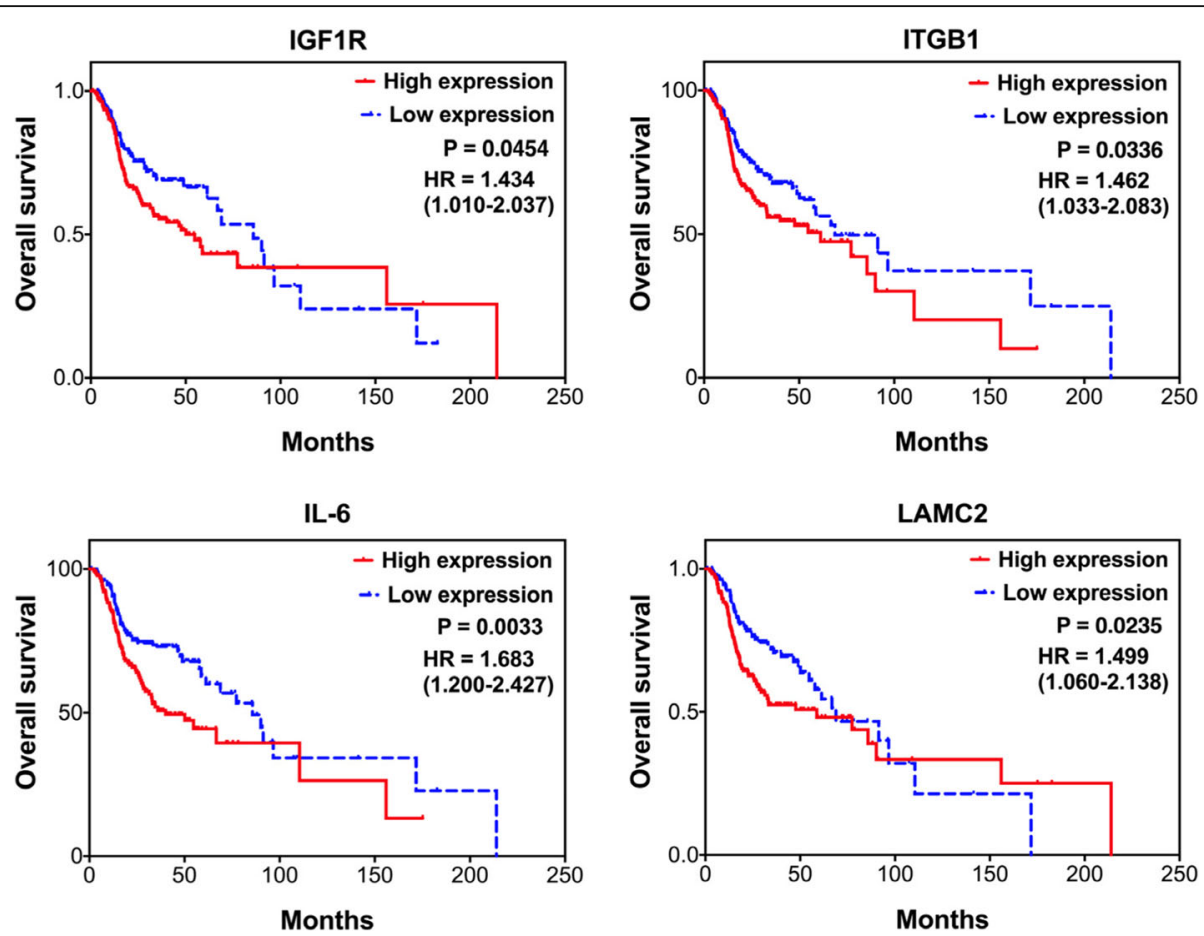

Fig. 4 Prognostic significance of the 4 key molecules (IGF1R, LAMC2, ITGB1 and IL-6) in HNC patients receiving radiotherapy, as determined by Kaplan-Meier survival analysis from the TCGA-HNSCC dataset $(n=502)$. The clinical data were retrieved via the Genomic Data Commons (GDC)Data-Portal of National Cancer Institute. For each gene, the survival curve, hazard ratio (HR) and $p$-value (P) are shown

signaling pathway is also an important factor contributing to radioresistance in many cancers [64, 65]. All these reports support our findings, suggesting that the intratumor hypoxia condition induces the HIF-1 signaling pathway, further facilitating cancer stemness formation and resulting in radioresistance in HNC. We also noted

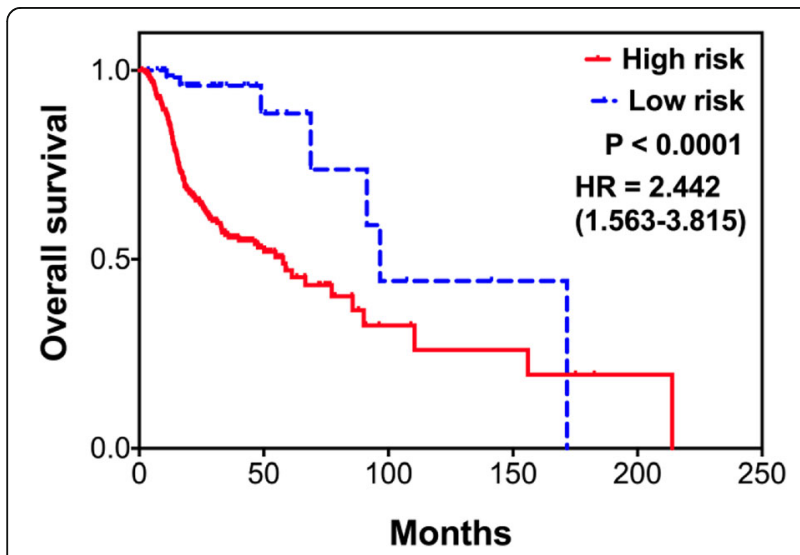

Fig. 5 Prognostic effectiveness of the combined 4 markers in HNC patients receiving radiotherapy, as determined by Kaplan-Meier survival analysis from the TCGA-HNSCC dataset $(n=502)$. The patients possessed at least one high-level markers were defined as the high-risk group, while those without any high-level marker being low-risk group. The survival curve, hazard ratio (HR) and $p$ value $(P)$ are shown that IGF1R is an important molecule participating in the HIF-1 signaling pathway and regulating cancer stemness (Tables 1 and 2). These results are consistent with previous studies by other investigators [66-69]. IGF1R has tyrosine kinase activity, which plays a significant role during cancer cell transformation mainly via cytoprotection and anti-apoptosis [70-72]. Apparently, high expression of this molecule in tumors confers resistance to chemo-radiotherapy [70-72]. Consistently, we found that IGF1R is over-expressed in cancer tissues and is associated with adverse outcomes in HNC patients receiving radiotherapy (Figs. 3 and 4). Similar results were also found in many types of cancers, including prostate, lung, ovary and renal cancers [73-77]. Taken together, our results demonstrated that IGF1R is an unfavorable prognostic marker in HNC patients treated with radiotherapy. This may result from modulation of the HIF-1 signaling pathway, leading to stem cell conversion and a radioresistant phenotype.

\section{Conclusions}

Multi-gene biomarker is one of the most important issues for clinical application. In this study, we have identified 255 genes significantly associated with radioresistance in HNC through global survey techniques. Via data mining and bioinformatic analyses, we have addressed 4 signaling pathways and 11 molecules associated with poor survival 
in HNC. Among these molecules, 4 markers LAMC2, ITGB1, IL-6, and IGF1R were demonstrated to form a panel marker to predict a worse radiotherapeutic outcome. This panel signature can be applied as predictive information for radiotherapy and may be further used to develop radio-sensitizing modalities for the treatment of refractory HNC.

\section{Additional files}

Additional file 1: Figure S1. Verification of the radioresistant phenotype of radioresistant sublines. A total of 500 cells parental (OECM1-Pt, Detroit-Pt, FaDu-Pt) or radioresistant subline cells (OECM1-RR, Detroit-RR, and FaDu-RR) were seeded per well in a 96-well plate and following treated irradiation with various doses $(0,3$, and $6 \mathrm{~Gy})$ and continuously cultured for 4 days. The cell survival fractions were assessed using Cell Counting Kit-8. Results were presented as the mean \pm standard deviation (SD) from three independent experiments. ${ }^{* * *}, P<0.0001$. Figure $\mathbf{S}$. Hierarchical clustering analysis of the gene expression profiles among the three HNC cell lines (OECM1, FaDu, Detroit) and their radioresistant (RR) sublines. Figure S3. Differential expressions of 4 marker proteins (IFGIR, LAMC2, ITGB1, and IL6) between HNC cell lines and their radioresistant sublines. Three HNC parental cells (OECM1-Pt, Detroit-Pt, FaDu-Pt) and their radioresistant subline cells (OECM1-RR, Detroit$R R$, and FaDu-RR) were used. The IGF1R, LAMC2, TGB1, and GAPDH were determined from total cell lysate, whereas IL-6 was determined from serumfree conditioned medium (CM). The relative expression of protein was normalized to GAPDH in each individual sample. Over-expression of these four proteins were found in the radioresistant subline cells as revealed by Western blotting analysis. Figure S4. Prognostic significance of the combined 7 RR molecules (IGF1R, LAMC2, ITGA6, ITGB1, ITGB4, LAMA3 and IL6) in HNC patients, as determined by SurvExpress analysis from two different TCGAHNSCC datasets ( $n=283$, and $n=502$ ). In each dataset, the log-rank test of the Kaplan-Meier survival curve, the hazard ratio (HR) and the $p$-value (P) are shown. (DOCX $1398 \mathrm{~kb})$

Additional file 2: Table S1. The top 100 up-regulated genes associated with radioresistance in HNC cells. Table S2. The top 50 down-regulated genes associated with radioresistance in HNC cells. Table S3. Correlative expressions of the 7 molecules in HNC patients receiving radiotherapy. (DOCX $42 \mathrm{~kb})$

\section{Abbreviations}

Cls: Confidence intervals; HNC: Head and neck cancer; HNSCC: Head and neck squamous cell carcinoma; HR: Hazard ratio; KEGG: Kyoto encyclopedia of genes and genomes; RR: Radioresistant; TCGA: The cancer genome atlas

\section{Acknowledgements}

Not applicable.

\section{Funding}

This project was supported by the grants from Chang Gung Memorial Hospital (CORPG3E0111, CORPG3G0931, CORPG3G0941, CPRPG3E0021 2). The role of the funding body JTC for these grants include the design of the study, collection, analysis and interpretation of data, and writing the manuscript.

\section{Availability of data and materials}

The mRNA expression of head and neck cancer tissues were mined from TCGA-HNSCC cohort (https://gdac.broadinstitute.org) and clinical information were available in the Genomic Data Commons Data Portal (National Cancer Institute, NIH, USA) repository. SurvExpress web tool generate Kaplan-Meier analysis and box plots from TCGA-HNSC datasets. All relevant materials are provided in the manuscript.

\section{Authors' contributions}

GRY, AJC and JTC participated the entire study, including the study design, data interpretation, result discussion, manuscript revision and finalization. GRY and AJC performed the data collection and was responsible for drafting of the manuscript. $L Y L, Y C H, Y J C$, and $H L$ contribute to study design, data analysis and manuscript finalization. All authors agreed to be accountable for all aspects of the work in ensuring that questions related to the accuracy or integrity of the work are appropriately investigated, and gave final approval of the revision to be published.

Ethics approval and consent to participate

The data of head and neck cancer patients we used in this study are all from the publicly accessible TCGA database. The TCGA offer anonymous data.

Consent for publication

Not applicable.

\section{Competing interests}

The authors declare that they have no competing interests.

\section{Publisher's Note}

Springer Nature remains neutral with regard to jurisdictional claims in published maps and institutional affiliations.

\section{Author details}

${ }^{1}$ Graduate Institute of Biomedical Sciences, College of Medicine, Chang Gung University, Taoyuan, Taiwan. ${ }^{2}$ Department of Medical Biotechnology and Laboratory Science, College of Medicine, Chang Gung University, Taoyuan, Taiwan. ${ }^{3}$ Department of Radiation Oncology, Chang Gung Memorial Hospital-Linkou, Taoyuan, Taiwan. ${ }^{4}$ Department of Pathology, Chang Gung Memorial Hospital-Linkou, Taoyuan, Taiwan. ${ }^{5}$ Department of Oral Maxillofacial Surgery, Chang Gung Memorial Hospital-Linkou, Taoyuan, Taiwan. ${ }^{6}$ Department of Biochemistry and Molecular Biology, College of Medicine, Chang Gung University, Taoyuan, Taiwan. ${ }^{7}$ Molecular Medicine Research Center, Chang Gung University, Taoyuan, Taiwan. ${ }^{8}$ Graduate Institute of Biomedical Materials and Tissue Engineering, College of Biomedical Engineering, Taipei Medical University, Taipei, Taiwan. ${ }^{9}$ School of Biomedical Engineering, College of Biomedical Engineering, Taipei Medical University, Taipei, Taiwan. ${ }^{10}$ Department of Radiation Oncology, Xiamen Chang Gung Memorial Hospital, Xiamen, Fujian, China.

Received: 3 August 2018 Accepted: 21 December 2018

Published online: 14 January 2019

\section{References}

1. Torre LA, Bray F, Siegel RL, Ferlay J, Lortet-Tieulent J, Jemal A. Global cancer statistics, 2012. CA Cancer J Clin. 2015;65:87-108.

2. Saman DM. A review of the epidemiology of oral and pharyngeal carcinoma: update. Head Neck Oncol. 2012;4(1).

3. Chen YJ, Chang JT, Liao CT, Wang HM, Yen TC, Chiu CC, et al. Head and neck cancer in the betel quid chewing area: recent advances in molecular carcinogenesis. Cancer Sci. 2008;99:1507-14.

4. Higgins GS, O'Cathail SM, Muschel RJ, McKenna WG. Drug radiotherapy combinations: review of previous failures and reasons for future optimism. Cancer Treat Rev. 2015;41:105-13.

5. Klein J, Livergant J, Ringash J. Health related quality of life in head and neck cancer treated with radiation therapy with or without chemotherapy: a systematic review. Oral Oncol. 2014;50:254-62.

6. Wong YF, Sahota DS, Cheung TH, Lo KW, Yim SF, Chung TK, et al. Gene expression pattern associated with radiotherapy sensitivity in cervical cancer. Cancer J. 2006;12:189-93.

7. Guo WF, Lin RX, Huang J, Zhou Z, Yang J, Guo GZ, et al. Identification of differentially expressed genes contributing to radioresistance in lung cancer cells using microarray analysis. Radiat Res. 2005;164:27-35.

8. Higo M, Uzawa K, Kouzu Y, Bukawa H, Nimura SN, et al. Identification of candidate radioresistant genes in human squamous cell carcinoma cells through gene expression analysis using DNA microarrays. Oncol Rep. 2005; 14:1293-8.

9. Fukuda K, Sakakura C, Miyagawa K, Kuriu Y, Kin S, Nakase Y, et al. Differential gene expression profiles of radioresistant oesophageal cancer cell lines established by continuous fractionated irradiation. Br J Cancer. 2004;91:1543-50.

10. Ogawa K, Utsunomiya T, Mimori K, Tanaka F, Haraguchi N, Inoue H, et al. Differential gene expression profiles of radioresistant pancreatic cancer cell lines established by fractionated irradiation. Int J Oncol. 2006;28:705-13. 
11. Chang JT, Chan SH, Lin CY, Lin TY, Wang HM, Liao CT, et al. Differentially expressed genes in radioresistant nasopharyngeal cancer cells: gp96 and GDF15. Mol Cancer Ther. 2007;6:2271-9.

12. Lin TY, Chang JT, Wang HM, Chan SH, Chiu CC, Lin CY, et al. Proteomics of the radioresistant phenotype in head-and-neck cancer: gp96 as a novel prediction marker and sensitizing target for radiotherapy. Int J Radiat Oncol Biol Phys. 2010;78:246-56.

13. Li YL, Chang JT, Lee LY, Fan KH, Lu YC, Li YC, et al. GDF15 contributes to radioresistance and cancer stemness of head and neck cancer by regulating cellular reactive oxygen species via a SMAD-associated signaling pathway. Oncotarget. 2017:8:1508-28.

14. De Llobet LI, Baro M, Figueras A, Modolell I, Da Silva MV, Munoz P, et al. Development and characterization of an isogenic cell line with a radioresistant phenotyhpe. Clin Trans Oncol. 2013;15:189-97.

15. McDermott N, Meunier A, Lynch TH, Hollywood D, Marignol L. Isogenic radiation resistant cell lines: development and validation strategies. Int J Radiat Biol. 2014;90:115-26.

16. Kuwahara Y, Roudkenar MH, Urushihara Y, Saito Y, Tomita K, Roushandeh AM, et al. Clinically relevant radioresistant cell line: a simple model to understand cancer Radioresistance. Med Mol Morphol. 2017;50:195-204.

17. Kang CJ, Chen YJ, Liao CT, Wang HM, Chang JT, Lin CY, et al. Transcriptome profiling and network pathway analysis of genes associated with invasive phenotype in oral cancer. Cancer Lett. 2009;284:131-40.

18. Kanehisa M, Goto S. KEGG: Kyoto encyclopedia of genes and genomes. Nucleic Acids Res. 2000;28:27-30.

19. Aguirre-Gamboa RG-RH, Martínez-Ledesma E, Martínez-Torteya A, ChacollaHuaringa R, Rodriguez-Barrientos A, et al. SurvExpress: an online biomarker validation tool and database for cancer gene expression data using survival analysis. PLoS One. 2013;8:e74250.

20. Patarroyo $M$, Tryggvason $K$, Virtanen I. Laminin isoforms in tumor invasion, angiogenesis and metastasis. Semin Cancer Biol. 2002;12:197-207.

21. Pandolfi F, Franza L, Altamura S, Mandolini C, Cianci R, Ansari A, et al. Integrins: integrating the biology and therapy of cell-cell interactions. Clin Ther. 2017;39:2420-36.

22. Belkin AM, Stepp MA. Integrins as receptors for laminins. Microsc Res Tech. 2000;51:280-301.

23. Tsuruta D, Kobayashi $H$, Imanishi $H$, Sugawara K, Ishii M, Jones JC. Laminin332- integrin interaction: a target for cancer therapy? Curr Med Chem. 2008; 15:1968-75.

24. Eke I, Deuse Y, Hehlgans S, Gurtner K, Krause M, Baumann M, et al. $\beta_{1}$ Integrin/FAK/cortactin signaling is essential for human head and neck cancer resistance to radiotherapy. J Clin Invest. 2012;122:1529-40.

25. Ou J, Luan W, Deng J, Sa R, Liang H. aV integrin induces multicellular radioresistance in human nasopharyngeal carcinoma via activating SAPK JNK pathway. PLoS One. 2012;7:e38737.

26. Yang D, Shi J, Fu H, Wei Z, Xu J, Hu Z, et al. Integrin $\beta 1$ modulates tumour resistance to gemcitabine and serves as an independent prognostic factor in pancreatic adenocarcinomas. Tumour Biol. 2016;37:12315-27.

27. Kyjacova L, Hubackova S, Krejcikova K, Strauss R, Hanzlikova H, Dzijak R, et al Radiotherapy-induced plasticity of prostate cancer mobilizes stem-like nonadherent, Erk signaling-dependent cells. Cell Death Differ. 2015;22:898-911.

28. Zutter MM. Integrin-mediated adhesion: tipping the balance between chemosensitivity and chemoresistance. Adv Exp Med Biol. 2007;608:87-100.

29. Kamada M, Koshikawa N, Minegishi T, Kawada C, Karashima T, Shulin T, et al. Urinary laminin- 22 is a novel biomarker of non-muscle invasive urothelial carcinoma. Cancer Sci. 2015;106:1730-7.

30. Korbakis D, Dimitromanolakis A, Prassas I, Davis GJ, Barber E, Reckamp KL, et al. Serum LAMC2 enhances the prognostic value of a multi-parametric panel in non-small cell lung cancer. Br J Cancer. 2015;113:484-91.

31. Huang D, Du C, Ji D, Xi J, Gu J. Overexpression of LAMC2 predicts poor prognosis in colorectal cancer patients and promotes cancer cell proliferation, migration, and invasion. Tumour Biol. 2017;39:1-7.

32. Patel V, Aldridge K, Ensley JF, Odell E, Boyd A, Jones J, et al. Laminingamma2 overexpression in head-and-neck squamous cell carcinoma. Int J Cancer. 2002;99:583-8

33. Gasparoni A, Della Casa M, Milillo L, Lorenzini G, Rubini C, Urso R, et al. Prognostic value of differential expression of Laminin-5 gamma2 in oral squamous cell carcinomas: correlation with survival. Oncol Rep. 2007;18:793-800.

34. Zhao ZS, Li L, Wang HJ, Wang YY. Expression and prognostic significance of CEACAM6, ITGB1, and CYR61 in peripheral blood of patients with gastric cancer. J Surg Oncol. 2011;104:525-9.
35. Yin HL, Wu CC, Lin CH, Chai CY, Hou MF, Chang SJ, et al. $\beta 1$ integrin as a prognostic and predictive marker in triple-negative breast cancer. Int J Mol Sci. 2016;17:1432.

36. Klahan S, Huang WC, Chang CM, Wong HS, Huang CC, Wu MS, et al. Gene expression profiling combined with functional analysis identify integrin beta1 (ITGB1) as a potential prognosis biomarker in triple negative breast cancer. Pharmacol Res. 2016;104:31-7.

37. Wang XM, Li J, Yan MX, Liu L, Jia DS, Geng Q, et al. Integrative analyses identify osteopontin, LAMB3 and ITGB1 as critical pro-metastatic genes for lung cancer. PLoS One. 2013;8:e55714.

38. Xheng W, Jiang C, Li R. Integrin and gene network analysis reveals that ITGA5 and ITGB1 are prognostic in non-small-cell lung cancer. Onco Targets Ther. 2016:9:2317-27.

39. Garg M, Braunstein G, Koeffler HP. LAMC2 as a therapeutic target for cancers. Expert Opin Ther Targets. 2014;18:979-82.

40. Poschau M, Dickreuter E, Singh-Müller J, Zscheppang K, Eke I, Liersch T, et al. EGFR and $\beta 1$-integrin targeting differentially affect colorectal carcinoma cell radiosensitivity and invasion. Radiother Oncol. 2015;116: 510-6.

41. Eke I, Cordes N. Focal adhesion signaling and therapy resistance in cancer. Semin Cancer Biol. 2015;31:65-75.

42. Babel L, Grunewald M, Lehn R, Langhans M, Meckel T. Direct evidence for cell adhesion-mediated radioresistance (CAM-RR) on the level of individual integrin $\beta 1$ clusters. Sci Rep. 2017;7:3393.

43. Martini M, De Santis MC, Braccini L, Gulluni F, Hirsch E. PI3K AKT signaling pathway and cancer: an updated review. Ann Med. 2014;46:372-83.

44. Toulany M, Rodemann HP. Phosphatidylinositol 3-kinase/Akt signaling as a key mediator of tumor cell responsiveness to radiation. Semin Cancer Biol. 2015:35:180-90.

45. Perri F, Pacelli R, Della Vittoria Scarpati G, Cella L, Giuliano M, Caponigro F, et al. Radioresistance in head and neck squamous cell carcinoma: biological bases and therapeutic implications. Head Neck. 2015;37:763-70.

46. Hunter CA, Jones SA. IL-6 as a keystone cytokine in health and disease. Nat Immunol. 2015;16:448-57.

47. Schaper F, Rose-John S. Interleukin-6: biology, signaling and strategies of blockade. Cytokine Growth Factor Rev. 2015;26:475-87.

48. Choudhary MM, France TJ, Teknos TN, Kumar P. Interleukin-6 role in head and neck squamous cell carcinoma progression. World J Otorhinolaryngol Head Neck Surg. 2016;2:90-7.

49. Yang L, Han S, Sun Y. An IL6-STAT3 loop mediates resistance to PI3K inhibitors by inducing epithelial-mesenchymal transition and cancer stem cell expansion in human breast cancer cells. Biochem Biophys Res Commun. 2014;453:582-7.

50. Smith DA, Kiba A, Zong Y, Witte ON. Interleukin-6 and oncostatin-M synergize with the PI3K/AKT pathway to promote aggressive prostate malignancy in mouse and human tissues. Mol Cancer Res. 2013;11:1159-65.

51. Chen Y, Zhang F, Tsai Y, Yang X, Yang L, Duan S, et al. IL-6 signaling promotes DNA repair and prevents apoptosis in CD133+ stem-like cells of lung cancer after radiation. Radiat Oncol. 2015;10:227.

52. Tamari $Y$, Kashino G, Mori H. Acquisition of radioresistance by IL-6 treatment is caused by suppression of oxidative stress derived from mitochondria after Y-irradiation. J Radiat Res. 2017;58:412-20.

53. Matsuoka Y, Nakayama H, Yoshida R, Hirosue A, Nagata M, Tanaka T, et al. IL-6 controls resistance to radiation by suppressing oxidative stress via the Nrf2-antioxidant pathway in oral squamous cell carcinoma. Br J Cancer. 2016;115:1234-44

54. Kumari N, Dwarakanath BS, Das A, Bhatt AN. Role of interleukin-6 in cancer progression and therapeutic resistance. Tumour Biol. 2016;37:11553-72.

55. Song Z, Lin Y, Ye X, Feng C, Lu Y, Yang G, et al. Expression of IL-1a and IL-6 is associated with progression and prognosis of human cervical cancer. Med Sci Monit. 2016;22:4475-81.

56. Liao C, Yu Z, Guo W, Liu Q, Wu Y, Li Y, et al. Prognostic value of circulating inflammatory factors in non-small cell lung cancer: a systematic review and meta-analysis. Cancer Biomark. 2014;14:469-81.

57. Zhang M, Gong W, Zhang Y, Yang Y, Zhou D, Weng M, et al. Expression of interleukin-6 is associated with epithelial-mesenchymal transition and survival rates in gallbladder cancer. Mol Med Rep. 2015;11:3539-46.

58. Jinno T, Kawano S, Maruse Y, Matsubara R, Goto Y, Sakamoto T, et al. Increased expression of interleukin-6 predicts poor response to chemoradiotherapy and unfavorable prognosis in oral squamous cell carcinoma. Oncol Rep. 2015;33:2161-8. 
59. Yang C, Jin K, Tong Y, Cho WC. Therapeutic potential of cancer stem cells. Med Oncol. 2015;32:619.

60. Skvortsova I, Debbage P, Kumar V, Skvortsov S. Radiation resistance: Cancer stem cells (CSCS) and their enigmatic pro-survival signaling. Semin Cancer Biol. 2015;35:39-44.

61. Peng G, Liu Y. Hypoxia-inducible factors in cancer stem cells and inflammation. Trends Pharmacol Sci. 2015;36:374-83.

62. Vadde R, Vemula S, Jinka R, Merchant N, Bramhachari PV, Nagaraju GP. Role of hypoxia- inducible factors (HIF) in the maintenance of stemness and malignancy of colorectal cancer. Crit Rev Oncol Hematol. 2017;113:22-7.

63. Ye J, Wu D, Wu P, Chen Z, Huang J. The cancer stem cell niche: cross talk between cancer stem cells and their microenvironment. Tumour Biol. 2014; 35:3945-51.

64. Harada H. Hypoxia-inducible factor 1-mediated characteristic features of cancer cells for tumor radioresistance. J Radiat Res. 2016;57(Suppl 1):i99-i105.

65. Vordermark D, Horsman MR. Hypoxia as a biomarker and for personalized radiation oncology. Recent Results Cancer Res. 2016;198:123-42.

66. Gariboldi MB, Ravizza R, Monti E. The IGFR1 inhibitor NVP-AEW541 disrupts a pro-survival and pro-angiogenic IGF-STAT3-HIF1 pathway in human glioblastoma cells. Biochem Pharmacol. 2010;80:455-62.

67. Murakami A, Takahashi F, Nurwidya F, Kobayashi I, Minakata K, Hashimoto $M$, et al. Hypoxia increases gefitinib-resistant lung cancer stem cells through the activation of insulin-like growth factor 1 receptor. PLoS One. 2014;9: e86459.

68. Chang WW, Lin RJ, Yu J, Chang WY, Fu CH, Lai A, et al. The expression and significance of insulin-like growth factor-1 receptor and its pathway on breast cancer stem/progenitors. Breast Cancer Res. 2013;15:R39.

69. Kuo YC, Au HK, Hsu JL, Wang HF, Lee CJ, Peng SW, et al. IGF-1R promotes symmetric self-renewal and migration of alkaline phosphatase+ germ stem cells through HIF-2a-OCT4/CXCR4 loop under hypoxia. Stem Cell Reports. 2018;10:524-37.

70. Motallebnezhad M, Aghebati-Maleki L, Jadidi-Niaragh F, Nickho H, SamadiKafil H, Shamsasenjan K, et al. The insulin-like growth factor-I receptor (IGFIR) in breast cancer: biology and treatment strategies. Tumour Biol. 2016;37: $11711-21$.

71. Yuan J, Yin Z, Tao K, Wang G, Gao J. Function of insulin-like growth factor 1 receptor in cancer resistance to chemotherapy. Oncol Lett. 2018;15:41-7.

72. Valenciano A, Henríquez-Hernández LA, Moreno M, Lloret M, Lara PC. Role of IGF-1 receptor in radiation response. Transl Oncol. 2012:5:1-9.

73. Zhao J, Shi X, Wang T, Ying C, He S, Chen Y. The prognostic and clinicopathological significance of IGF-1R in NSCLC: a meta-analysis. Cell Physiol Biochem. 2017;43:697-704.

74. Alelsic T, Verrill C, Bryant RJ, Han C, Worrall AR, Brureau L, et al. IGF-1R associates with adverse outcomes after radical radiotherapy for prostate cancer. Br J Cancer 2017; 117: 1600-1606.

75. Amutha P, Rajkumar T. Role of insulin-like growth factor, insulin-like growth factor receptors, and insulin-like growth factor-binding proteins in ovarian cancer. Indian J Med Paediatr Oncol. 2017;38:198-206.

76. Solarek W, Czarnecka AM, Escudier B, Bielecka ZF, Lian F, Szczylik C. Insulin and IGFs in renal cancer risk and progression. Endocr Relat Cancer. 2015;22: R253-64.

77. Dale OT, Aleksic T, Shah KA, Han C, Mehanna H, Rapozo DC, et al. IGF-1R expression is associated with HPV-negative status and adverse survival in head and neck squamous cell cancer. Carcinogenesis. 2015;36:648-55.

\section{Ready to submit your research? Choose BMC and benefit from:}

- fast, convenient online submission

- thorough peer review by experienced researchers in your field

- rapid publication on acceptance

- support for research data, including large and complex data types

- gold Open Access which fosters wider collaboration and increased citations

- maximum visibility for your research: over $100 \mathrm{M}$ website views per year

At BMC, research is always in progress.

Learn more biomedcentral.com/submissions 